\title{
Vehicles for Open-Pit Mining with Smart Scheduling System for Transportation Based on $5 G$
}

\author{
Mohanad F Jwaid ${ }^{1}$, Husam K Salih Juboori ${ }^{2}$ \\ ${ }^{1}$ Assistant Lecturer, Al Immam University College, Balad, Iraq. \\ ${ }^{2}$ Pharmacy Department, Al Rasheed University College, Baghdad, Iraq. \\ $\underline{\text { mohanad02jwaid@gmail.com }}^{1}, \underline{\text { husam01salih@gmail.com }}^{2}$
}

Article History: Received: 11 January 2021; Accepted: 27 February 2021; Published online: 5 April 2021

\begin{abstract}
In the Recent times, various technological enhancements in the field of artificial intelligence and big data has been noticed. This advancements coupled with the evolution of the $5 \mathrm{G}$ communication and Internet of Things technologies, has helped in the development in the domain of smart mine construction. The development of unmanned vehicles with enhanced and smart scheduling system for open-pit mine transportation is one such much needed application. Traditional open-pit mining systems, which often cause vehicle delays and congestion, are controlled by human authority. The number of sensors has been used to operate unmanned cars in an open-pit mine. The sensors haves been used to prove the real-time data in large quantity. Using this data, we analyses and create an improved transportation scheduling mechanism so as to optimize the paths for the vehicles. Considering the huge amount the data received and aggregated through various sensors or sources like, the GPS data of the unmanned vehicle, the equipment information, an intelligent, and multi-target, open-pit mine unmanned vehicle schedules model was developed. It is also matched with real open-pit mine product to reduce transport costs, overall unmanned vehicle wait times and fluctuation in ore quality. To resolve the issue of scheduling the transportation, we prefer to use algorithms based on artificial intelligence. In addition to four other models we are proposing a decomposition-based restricted genetic dominance (DBCDP-NSGA-II) algorithm, which retains viable and non-facilitating solutions in small areas in order to improve the convergence, distribution and diversity of traditional high-dimensional multi-objective fast-dominated genetic sorting Algorithms (NSGA-II).

Keywords: 5G; Open-Pit Mine; Unmanned Driving; Traffic Big Data; Smart Scheduling; Intelligent Transportation System.
\end{abstract}

\section{Introduction}

Factors such as numerous workflow sections, tough environments and diverse working conditions constrain open pit mines. Unmentioned development is an obstacle to intelligent and unmentioned mining and current open-pit exploration is still in its infancy. Unmentioned production. Figure 1 also shows vehicles waiting in line and decreases output efficiency significantly [1]. In order to boost the performance of vehicle operations, multiple variables such as engines, ear rates, grinding stations, ear material, etc. must be considered in depth in order to schedule the operation of vehicles

Itineraries (Figure 2). The Rio Tinto Group is a world-leading mining and automated drive company [2]. Rio Tinto In 2018, the Rio Tinto Group was the first unmanned vehicle batch to operate in 1,700 kilometers, raising the speed of unmanned iron oil transportation vehicles by 6 percent and decreasing the effect of shifting drivers [3].

A Global Positioning System (GPS) system is being introduced by Komatsu, loading a large unmanned 100-ton mining dump truck and testing on Kalimantan Island [4]. An underground contact system for study has been developed at Stobie Mine by Canadian International Nickel. The scrapers, boiling plants and underground cars of the mine were all unknown, and employees operate the machines remotely on the site. No need to set up underground workers [5] is essentially required. A PITRAM Web-based online remote mining application system [6] has been created by Australian Micromine to reduce mining costs by 10 percent.

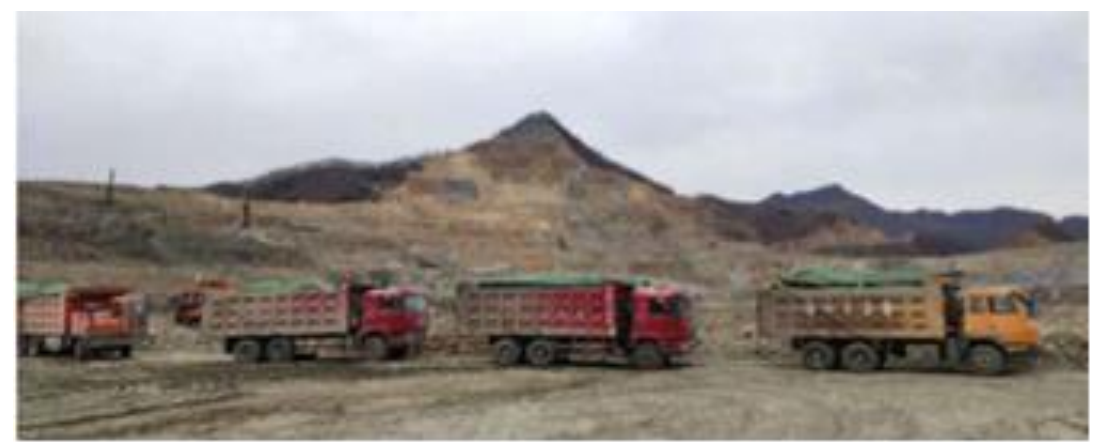

Figure 1. Vehicles in Queue for unloading. 


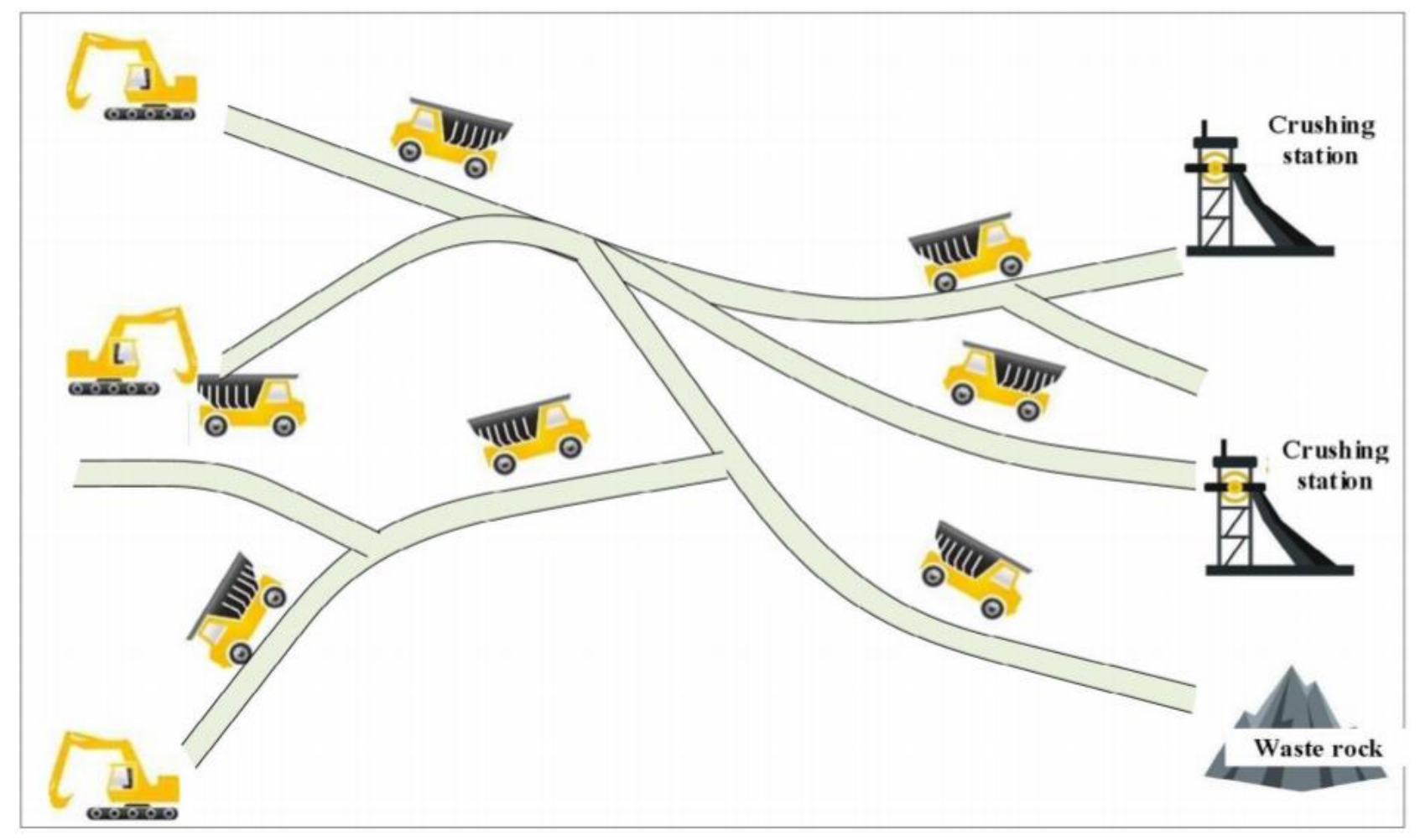

Figure 2. An Abstract view of Open-Pit Mine Vehicle Scheduling Model.

With the advancements in various technologies, it is evident that it will make an impact on Open-pit unknown driving technology. 5G technology, advanced data analytics, big data, artificial intelligence and other developments have a huge contribution in improvising the throughput. Centrally managed cloud storage and long-term networking can lead to a latency of the network and data cannot be reached quickly enough to satisfy user demands in real time, particularly under late critical circumstances [8]. Multiple sensors which constantly capture and rely on external information for unmanaged automobiles. Every few seconds, unmanned vehicles can produce GB-level data that presents challenges in measuring and storing the vehicle itself. The need for accurate data reply [9] is strong for unipersonal driving in open pit mines. The $5 \mathrm{G}$ network offers a secure signal link in open pit mines [10] compared to $4 \mathrm{G}$ and Wi-Fi.

5G high speed, highly precise guidance, low latency and routing, road scheduling, scheduling of activities, motion tracking, fusion data and other edge technologies can be deployed[11]. These systems have heavy running equipment specifications and cannot be accurately calculated on limited-resource mobile devices [12]. It is an efficient method for migration through computational migration technology to rich areas of resources or remote clouds for certain computationally complex multi-target optimizing algorithms [7]. Smart manufacturing and operating management for a mine open-pit unmanned truck dispatching device will help with the powerful computing capacity of edge computing [13]. The open-pit scheduling system requires, as shown in Figure 3, a collection of network computer nodes (service, base station, mobile terminal, user computer, different monitoring terminals, etc.). Unmanned scheduling system. In edge computation scenarios, the contact bandwidth between different nodes is very narrow and heterogeneous and multiple device nodes support a range of computational capabilities [14]. Edge computing connections connect directly or indirectly between network computing devices and nodes, greatly reducing remote communication time.

Data can be processed locally in a decentralized network as well as other regional nodes [15]. Network nodes can perform functions like offloading computation, caching and processing data and management of mobility [16]. The integrated 5G, BDC and artificial intelligence algorithms allow independent driving and independent path design for open-pit driverless vehicles. This means the use of 5G. Non-linear equation systems (NES) are used in many fields including electricity systems, machining, neural networks, design recognition, planning of output, network communication, portfolios of investment, image processing, etc. The solution of NESs has therefore become a very interesting subject for science. This paper poses fundamentally a NES problem for the car scheduling of vehicles (multi objective optimisation problem). 


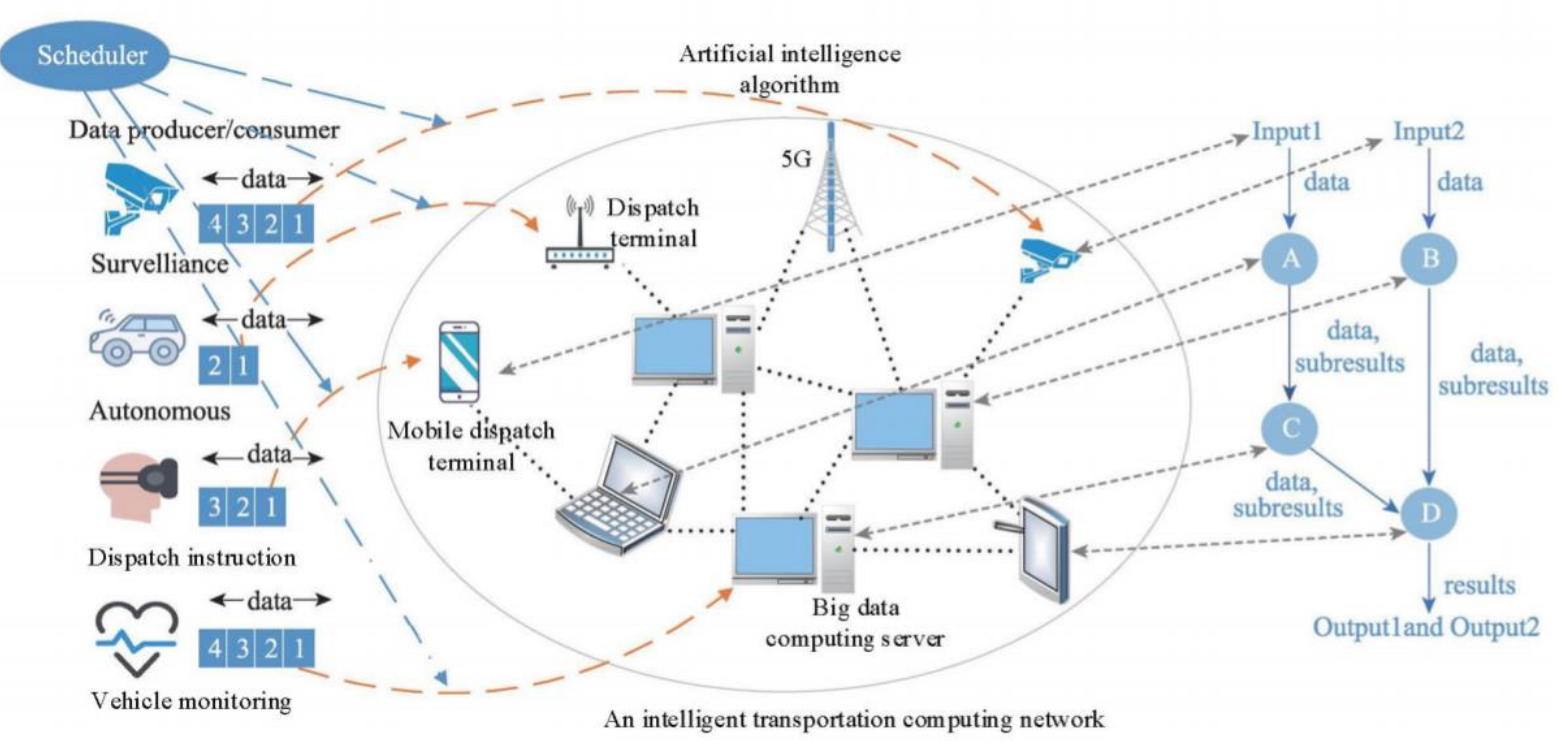

Figure 3. 5G Based open-pit mine vehicle scheduling system.

Many other NES solving algorithms are currently available and can be easily separated into two types: traditional optimisation and smart optimisation. The conventional streamlined NES solving algorithms generally provide incremental methods that are based on gradient experience, such as: gradient variations, Newton's method, pseudo Newton's, steepest method of descent etc. These processes depend on the selected initial stage. The root cannot be identified if the initial point is not picked correctly. Because of the need for gradient knowledge, it is only feasible to apply differential functions and quickly obtain an optimum local solution. More specifically, only one root in a single run can be found. The intelligent optimization technique is a group-based optimization method that concurrently scans and does not visibly parallel many locations. And the original specifications are not solid and are still true in non-differentiable NESs. The solution set is broad, efficient and robust. This simplifies the NES approach and replaces conventional optimization algorithms. Therefore, the use in recent years of intelligent evolutionary algorithm to solve NESs has become a place of research for both home and international students. In response to the NESs, Gao et al.[17] have conducted a hybrid evolutionary TPEA algorithm. The NESs are translated into single object evolutionary algorithm in this algorithm. In the first object, the NCDE niche helps to create an index of diversity based on the Gaussian kernel feature to maintain population diversity in order to achieve a balance between redistribution and diversity. In unique iterations, NCDE and NSGA-II produce alternatively high quality candidate solutions. The second stage consists of finding promising areas (including areas in which an optimal solution can be found) and identifying the NES root as a local search algorithm by DE (differential evolution).

Gong et al.[18] suggested a weight-based bi-target optimization algorithm (A-Web) using MONES on a more efficient basis (multi objective optimization of nonlinear equation systems). This algorithm randomly produces the weight of the goal function from 0 to 1 . There is a mixture of two search algorithms, SHADE (success history adaptation parameter) and NSGA-II to create offspring by mutation. During this method, the parameters are adapted to improve search precision. The preference of the person depends on the ranking and is not dominated.

The HCMOIWO (hybrid cooperative IWO) algorithm [19] was introduced by Ojha. For each subpopulation study, the group in such a system is divided into two identical communities each of which corresponds to an objective feature based on IWO and STS that incorporates all subpopulations. Certain experiments focused on their randomness in terms of optimization of open pit mines, transmitting and organizational hypotheses by simulation and queuing modelling theory and problem assessment. The theory of queuing was included. Gu Q etal [20] recommended the simulation model for the computer to track the optimization effects of the open pit mining paradigm statistical programming and to conclude that the inclusion of vehicles would not inherently optimize exploration and production.

Many other analysis is currently focused on the forecasting brand on open-pit mine schedules, while less popular is background information on the multi-target sophisticated dispatch model. Very frequently, the new approach to multi-target vehicles focuses on two objective functions with the least exceptions, including sales, travel costs or utilization of vehicles. The grade is used as a determine appropriate, even though rating limit will easily lead to optimization process of a small or zero number. There have however not been a range of realistic concerns addressed in the new multi-target path optimization model. The aim of this article will be vehicle costs, minimal overall queuing time and minimum uncertainty as a goal in the light of the above issues, the use of an autonomous cargo unit to save costs and increase performance in open-pit mining companies and to satisfy 
multi-target vehicle scheduling criteria. This article is available in the following language. In order to intelligently shipping new driverless, open-pit mining vehicles, a de-composure-dominated model was developed using the updated NSGA-II measurement process. Through demonstrations, it has been proved that the presented scheduling algorithm seems effective in addressing the issues like variations in mineral grade, reduction in transport costs and waiting time.

Any of the above methods for the overall programming support our planner, however. However the pace of this study is discussed. This paper's argument can be summarized in the following points:

A new programming paradigm is implemented to improve the productivity of cellular customers. The suggested algorithm applies to the same percentage parser. In particular, it concentrates on the hypothetical cell-centered user replacement with a cell-linked user in RBs as a way of dynamically allocating changes through RBs in compliance with the sample concept without enabling cell-linked users to write or use RBs. This risk could however decline if vital RBs was assigned to cellular-edge users with low signal strengths to avoid bad system usage.

We have a low degree of difficulty and are ideal for all TTIs. Our proposed scheduler aims at increasing cellular efficiency while maintaining the necessary performance to continue to achieve an acceptable level of performance for cell-centered users.

\section{Optimization Model For Scheculing}

In order to improve the performance of cellular end users or users with poor channel conditions, a new programming strategy is implemented. The algorithm introduced is an expansion of the relative comparable scheduler. The focus is on replacing the cell-centered user with a cell-based user in such RBs where the probability varies that RBs are used to prepare and use those RBs. This risk could gracefully decline to avoid inadequate infrastructure usage when large RBs are delegated to cellular end users with poor signal quality.

This can be made available by network providers to all smartphone users or to a limited subsidiary of cellular users under certain conditions (such as fees in exchange). Therefore a central network controller classifies one device as a feature (FU), which indicates a user, through checking for two requirements at a TTI, who is willing to benefit from the proposed scheduler. You should summaries the following. The conditions are as follows.

Criteria for FU grading:

1. The classification of consumers between the cell center and the cell edge is very important. When a consumer's case is below a threshold, it is known as a cellular limit. As presented in [21], out of total cell throughput this presents the fifth percentile point. Also, to measure immediate consumer performance CQI reports are used. Just these customers are responsible for improved demands for service.

2. For the improved facility, the client will pay additional charges:

As in [22, 24], operators can prefer to equate the system performance level provided to users with the price, according to their recorded RB use. Today, most operators use models that fix the constant rental prices of customers per time or per bit. This fits well in designing projects that enact blind fairness for users / communications without prioritizing users with deteriorated channel conditions (e.g. cell-edge users). In order for operators to balance usable customers with unfeatured users (who obtain more throughput privileges and additional RBs), they can use their proposed timetable technologies using different pricing structures (who lose some resources).

The suggested solution uses two steps of planning. The PF processing is done to define a PF customer, who are using the RB during the first stage. A second step of the schedule would then possibly decide whether the preferred PF recipient or one of the FU is allocated this RB. In comparison to the conventional PF schedule[24] for RB candidates based on the ratio from the existing instantaneous and cumulative average user performance, we have a second round to include the chosen PF user with any signal strength FUs (cell-based customers). The alternative between PF and FU is likely to be nuanced in terms of the choices to reconcile improved cell-based user productivity and overall system performance. We first define the particulars of our proposed algorithms in the following manner.

- The set of cell FUs is presented as $\left\{\mathrm{FU}_{1}, \mathrm{FU}_{2} \ldots, \mathrm{FU}_{\mathrm{N}}\right\}$, where $\mathrm{N}$ corresponds to the cell's number of FUs.

- The available cell RBs set per TTI are indicated as $\left\{\mathrm{RB}_{1}, \mathrm{RB}_{2}, \ldots, \mathrm{RB} \mathrm{B}_{\mathrm{M}}\right\}$, with $\mathrm{M}$ being the cell number of $\mathrm{RBs}$.

- The appropriate minimum chance of choosing the RB1 PF candidate is indicated as P(PF). This is a design parameter that the network operator can define. $\mathrm{P}[\mathrm{PF}]$ will be analysed later in order to assess the overall system performance.

- PF_C is indicated as the initial number of chances of an RB1 PF consumer.

- FU_Ci is indicated as the initial number of chances for RB1 FUi.

The total number of RB1 odds could therefore be defined as:

$U E_{-} C=P F_{-} C+\sum_{n=1}^{N} F U_{-} C_{i}$ 
We presume that any FU would have the same k Clusters as the first RB chances (i.e., RB1). In other terms, at first,

FU_C $\mathrm{C}_{\mathrm{i}}=\mathrm{K} ;$ for $\mathrm{i}=1 ; 2 ; \ldots ; \mathrm{N}$

We describes instructions for selecting the $\mathrm{K}$ scaling factor in order to achieve the best results. Therefore it is straightforward to see that the initial likelihood to pick a PF candidate for the first RB (i.e. RB1) is given by PF_C/(PF_C $+\mathrm{NK})$ as the probability of a user's selection is determined by dividing this number of opportunities by the total number of chances for that user.

Since the minimum initial likelihood to pick a PF consumer (for RB1) is $\mathrm{P}(\mathrm{PF})$, the following criterion should be fulfilled in terms of the number of opportunities:

$\frac{\left(P F_{-} C\right)}{\left(P F_{-} C+N K\right)}>=P(P F)$

The above means that PF_C, the original PF consumer probabilities at RB1, is set according to:

$$
P F_{-} C>=\frac{(P(P F) \times N \times K)}{(1-P(P F))}
$$

During the first RB, the PF and FU candidates are chosen according to their chances of initialization, according to (1) and (3), respectively. For each RB, the possibilities of inclusion are updated to the non-replacement sampling methodological approach. In particular, in one RB, the consumer chosen is either given a lower chance / likelihood, or even entirely eliminated from competition for the next RB, etc. As a result, the next RBs provide more options for users not chosen for an $\mathrm{RB}$. If all chances (PF_C and all FU_Ci) are zero, PF_C and FU_Ci respectively are reset to (1) and (3). The loop goes on until users use all RBs.

The general algorithm for programming can be summarized below (for each cell at the beginning of each TTI).

\section{Among all UEs in the cell, identify the featured set \{FU1,}

FU2, .., FUN\} based on the FU classification criteria.

2. Set the initial chances PF_Cand $F_{-} C_{i}($ for $1<=i<=N)$ according to (1) and (3), respectively.

3. For each RB1, RB2, ..., RBM, do:

a. Invoke the PF scheduler to select a PF user.

b. Create the set $U$ of users containing PF_Ccopies of the

PF user, and $F U_{-} C_{i}$ copies of $F U_{i}($ for $1<=i<=N)$.

c. Select one user from $U$ according to a uniform distribution, to utilize the RB.

d. Update the selection chances as follows:

- If the selected user is a PF user, then $P F_{-} C:=P F_{-} C-1$;

- If the selected user is $F U_{i}($ forany $1<=i<=N)$, then

$F U_{-} C_{i}:=F U_{-} C_{i}-1$.

e. If all chances (forPFandallFUs) reach zero, reset PF_Cand

$F U_{-} C_{i}($ for $1<=i<=N)$ according to (1) and (3),

respectively.

It ensures the use of additional RBs to boost their efficiency and prevent throttling by users who have historically been subject to lower instantaneous rates (including cell-edge operators). The stolen PF users RBs are eventually replaced by other cell users who have increased performance dynamically so that the allocated bandwidth is not seriously affected. This goal was upheld and illustrated, as seen in the section results and review.

\section{Results And Analysis}

In the system-level simulator Vienna LTE, the proposed algorithm is built on top of MATLAB. LTE Release-8 supports the used bandwidth scheme and carrier frequency. The other parameters have been chosen to be consistent and to be applicable to the device bandwidth used in a practical environment. We believe that all cellbased users can be treated as FUs without losing generality. If any cellular users fail to pay for better output in compliance with the additional price, this would mean a reduction in the number of controlled users. Therefore the average throughput for the other users is even higher than the result in the next segment in each group (cellcentric and cell-bound).

\subsection{Analysis of the initial scaling factor}

That the very first analysis takes the maximum valuation on initial openings in the EUFS algorithm (K). Our empirical method is based on studies with a wide range of $\mathrm{K}$ values and selects the best quality for the other 
observations. Application K depends on the performance of cell users, although in the following findings the influence of the EUFS method is thoroughly investigated for cellular centers users. The fig. 4 shows average cellular-edge consumer performance (on the $\mathrm{y}$ - axis) using specific $\mathrm{K}$ value (on the $\mathrm{x}$-axis) as opposed to their static probability average outputs. However according (1), $\mathrm{K}$ is the first chance for each cell-edge recipient (i.e., FU Ci).

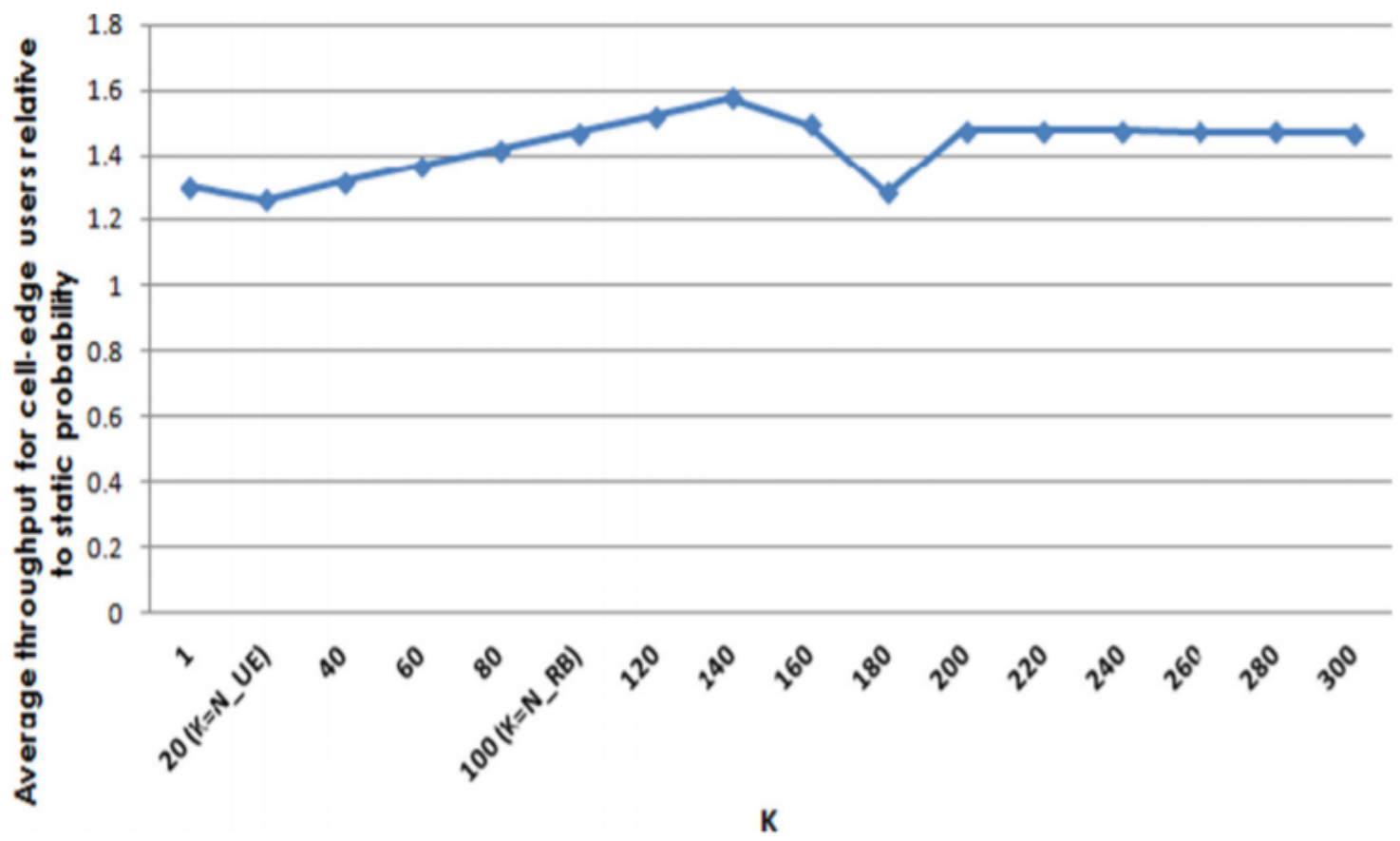

Figure 4. Average performance in relation to EUFS for cell-side users using fixed probabilities across different Ks in relation to average performance for cell-edge.

In order to preserve a fixed initial likelihood for $\mathrm{PF}$ user $\mathrm{P}(\mathrm{PF})$ during the $\mathrm{K}$ adjustment, the scaling $\mathrm{K}$ indicates an effective PF user scaling (i.e., PF C). In the first RB0, $\mathrm{P}(\mathrm{PF})$ is the only effective factor for the cell-edge or cell-centric consumer assignment of RB0. The option of $\mathrm{K}$ will take place in the following RBs, however, as there are various possibilities for adaptation after each RB. Small $\mathrm{K}$ values (i.e., $\mathrm{K} \ll<\mathrm{RBs}$ ) mean that the total number of odds for the various RBs is suddenly modified. It will really ensure that the modification of cell based consumers through one RB to another is assured, the algorithm will rapidly run out of opportunities and will reinitiate the odds of the chances outlined in EUFS section (e) all because the total chances (e.g. UE C) are directly proportional to $\mathrm{K}$. While it will guarantee the change in likelihood. Thus, due to the very regular reset of the total number of RBs, static likelihood is effectively performed. As shown in Fig., still with $\mathrm{K}=1$.

The assignment of dynamic probabilities by EUFS increases efficiency by about $20 \%$ over static probabilities. The machine memory for small $\mathrm{K}$ values is shallow which means a more fixed likelihood behaviour. During K, the deeper memory is retained and the users are allocated more adaptive RB. A better result is then obtained. And the opposite extreme case of $\mathrm{K}$ raises the value of RBs (i.e. $\mathrm{K}$ >> RBs) means that any application (interface or device user) has far too much initial chance. This further limits the efficiency of the hierarchical probability algorithm (EUFS), since only a single chance is excluded for each RB from all probabilities. For a very large overall likelihood, the influence of dynamic probability improvements by RBs is small and the method is closer to something like the constant likelihood allotment once again. The illustration. 4 shows that $\mathrm{K}=140$ achieves the optimum efficiency. We therefore decide not to break from the key goals of these experiments, but to take this value of $\mathrm{K}$ for the EUFS algorithm into consideration in the following experiments. In other words, the proposed algorithm is to be contrasted with modern algorithms while retaining a fixed scaling factor.

\subsection{Assessing the Throughput}

Then the average output is evaluated by all cell-centered users thus reducing the likelihood of PF candidates. As seen in the diagram. 5, for cell dependent users, the mean output of a pure PF scheduler (on y axis) sub linearly decreases as the chance assigned to the PF candidate decreases (on the $\mathrm{x}$-axis). By eliminating the potential for PF applicants, consumers would be given additional RBs and cell centers than a pure PF planner. The emerging EUFS optimization ensuring that the amount of RBs generated for cellular edge users from traditional PP candidates is not such a deteriorating factor as those RBs are proposed protocol drawn to make sure that losses are not focused on one or a few users that may cause their account to deteriorate. It can also be seen in the figure. 5 that the highest drop in cell-centric consumer performance for PF candidates happens at the lowest chance. 


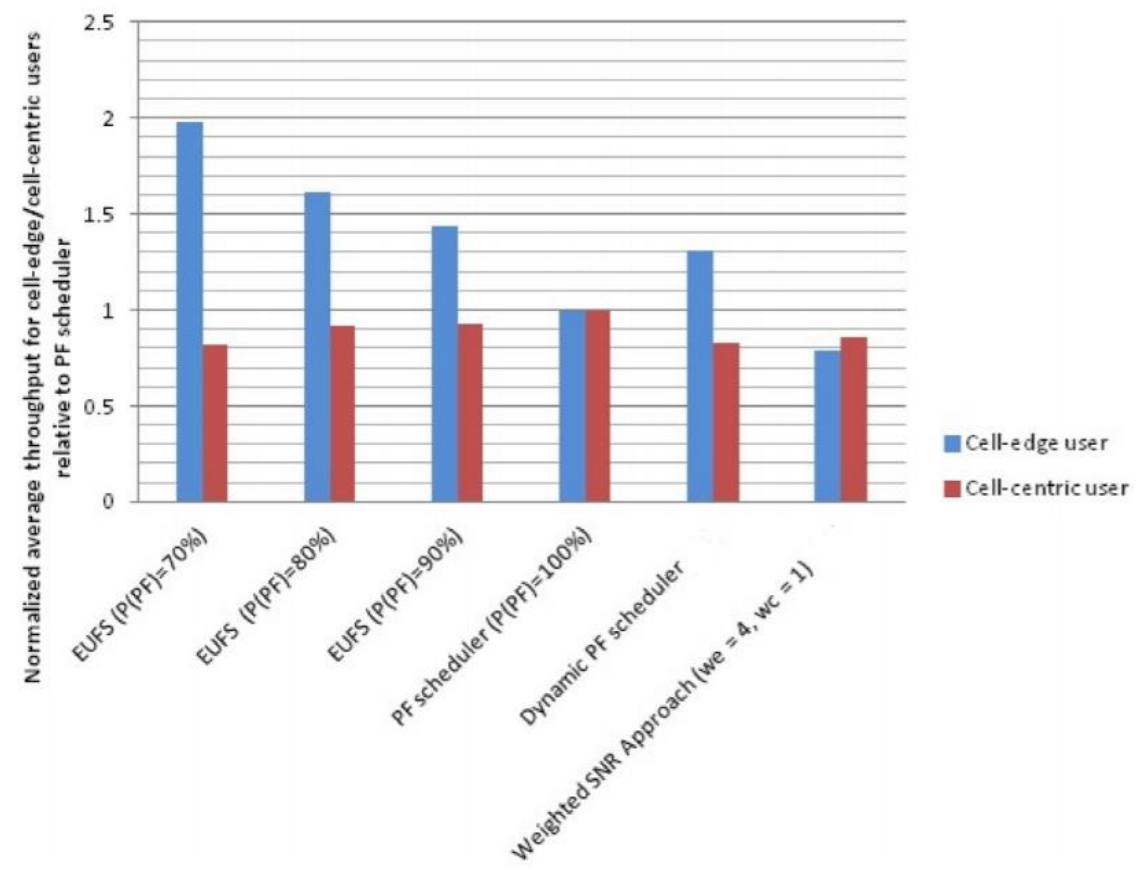

Figure 5. Comparative analysis for cellular/cellular users with various scheduling algorithms and fixed user numbers.

With regard to the EUFS algorithm, the cell-centered user performance drop by approximately $25 \%$ was set by the first likelihood for PF candidates at $82 \%$. As the likelihood of PF candidates is increased above 70 percent, this reduction is reduced to around 10 percent. The best compromise is to allocate probabilities to pf candidates from 80 percent to 90 percent by analyzing the output of both cell and cell center users while adjusting the likelihood of PF candidates. This raises the cell-edge user convergence speed to about 150 percent, while reducing the cell-centered user convergence speed by just about 10 percent. Operators might opt to further boost cell-side efficiency for the cell-centered user's factors affecting the performance for extended lengths of time. For this function, operators could use greater likelihood in experiments for PF candidates.

Our proposed approach is already above weighted SNR [15] algorithm. It is even higher than seen in Figure 5 for all PF probabilities. Our algorithm prevents users from getting a significant effect, when switching from any RB to the next, by readjusting the probabilities. The weighted SNR algorithm however doesn't preserve the adaptability necessary by using fixed weights in TTI, causing an aggressive user distribution of the RBs. When monitoring the average cell-centered user output as seen in the image, this behaviour is repeated. 5 .

In addition, Fig. 5 shows that in all cases of PF probabilities used, our proposed EUFS exceeds the dynamic PF algorithm [16]. The modification of user schedule priorities based on averaging user SINR, which is less effective in dynamic PF than using instantaneous user flow, as used in the algorithm proposed. Eventually, the initial solution of a considered mobile user (functional user) has been observed for various user counts. To that end, we hold the original possibilities of the PF nominee at $80 \%$.

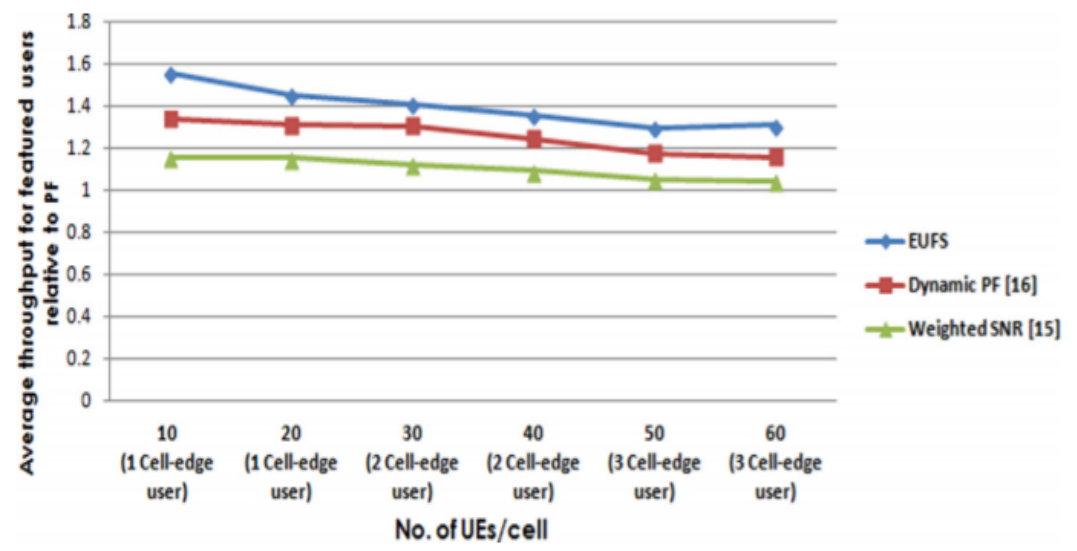

Figure 6. Comparative analysis for cellular devices using different scheduling scheme including variable users 
The illustration. 6 shows the average cell-side user per PF scheduler in various scheduling techniques (y-axis) as compared with the number of cell-side users (X-axis) per cell. 6 shows the average cell-side user performance. Other algorithms in all EU number/cell values are superior to our suggested EUFS algorithm. If we compare EUFS with the PF algorithm, we could observe a higher increase in the mean throughput at less UEs/cells, as compared to the higher UEs/cells ratio. For example, the average cell performance of 10 UEs / cell is about 150 percent compared to PF. For cell-edged users with EUFS algorithms. At $60 \mathrm{UEs} / \mathrm{cell}$, the average performance for cell-end users using EUFS algorithms is just about 125 percent in comparison with PF. As previously explained, the EUFS algorithm means that cell-based users have more RBs compared to the PF scheduler, which contribute to their achieved efficiency.

The figure. 6 also indicates that perhaps the methodology suggested by us is stronger than that of the weighted SNR algorithm, since EUFS implies a complex likelihood of switching between RBs by changing the chance. However the TI uses fixed weights in the weighted SNR algorithm which do not adjust and which forces users to constantly spread the RBs. Moreover, Fig. 6 demonstrates that the proposed UEFS algorithm exceeds the dynamic $\mathrm{PF}$ algorithm as it tends to be less efficient in the user's average PF scheduling expectations than instant user performance as is used in this proposed algorithm.

\section{Conclusion}

In contrast to the PF planner used in LTE, the proposed algorithm has demonstrated better efficiency for cell users. Moreover the side effects of a decrease in RBs that they are supposed to follow in PF technology have been shown to be limited on cell-centric users' efficiency. For featured and unsuited users, a comparison of results with LTE's leading scheduling technique was quantitatively assessed. Simulations show that the average effective way to boost the aggregate success of advantageous consumers is to give PF applicants a likelihood of 80 percent to 90 percent. As a result, the average non-beneficial consumer performance is only reduced to around $10 \%$. Our COMP and joint scheduling strategies may further benefit from our algorithm in order to improve device efficiency among all users.

\section{References}

1. B. Li, J. Li, K. Tang, and X. Yao, "Many-objective evolutionary algorithms: A survey," ACM Comput. Surv., vol. 48, no. 1, pp. 1-35, Sep. 2015.

2. Y. Zeng, J. Xie, H. Jiang, G. Huang, S. Yi, N. Xiong, and J. Li, "Smart caching based on user behavior for mobile edge computing," Inf. Sci., vol. 503, pp. 444-468, Nov. 2019.

3. J. B. Mendes, M. F. S. V. D'Angelo, N. A. Maia, and R. R. Veloso, "A hybrid multiobjective evolutionary algorithm for truck dispatching in Open-Pit-Mining," IEEE Latin Amer. Trans., vol. 14, no. 3, pp. 13291334, Mar. 2016.

4. Y. Liu, A. Liu, T.Wang, X. Liu, and N. N. Xiong, "An intelligent incentive mechanism for coverage of data collection in cognitive Internet of Things," Future Gener. Comput. Syst., vol. 100, pp. 701-714, Nov. 2019.

5. Z. Fan, W. Li, X. Cai, H. Li, C. Wei, Q. Zhang, K. Deb, and E. Goodman, "Push and pull search for solving constrained multi-objective optimization problems," Swarm Evol. Comput., vol. 44, pp. 665-679, Feb. 2019.

6. S. Jiang, M. Lian, C. Lu, S. Ruan, Z.Wang, and B. Chen, "SVM-DS fusion based soft fault detection and diagnosis in solar water heaters," Energy Explor. Exploitation, vol. 37, no. 3, pp. 1125-1146, May 2019.

7. Shahzad, J.-Y. Choi, N. Xiong, Y.-G. Kim, and M. Lee, "Centralized connectivity for multiwireless edge computing and cellular platform: A smart vehicle parking system," Wireless Commun. Mobile Comput., vol. 2018, pp. 1-23, 2018.

8. K. Gao, D. Yan, F. Yang, J. Xie, L. Liu, R. Du, and N. Xiong, "Conditional arti cial potential eld-based autonomous vehicle safety control with interference of lane changing in mixed traf c scenario," Sensors, vol. 19, no. 19, pp. 4199-4212, Sep. 2019.

9. W. Zhang, D. Chen, H. Si, and N. N. Xiong, "RTDCM: A coding preemption collection system for key data prioritization with hierarchical probability exchange mechanism in mobile computing," IEEE Access, vol. 8, pp. 4629-4639, 2019.

10. G. S. Bastos, "Decision making applied to shift change in stochastic open-pit mining truck dispatching," IFAC Proc. Volumes, vol. 46, no. 16, pp. 34-39, 2013.

11. X. Nie, S. Feng, Z. Shudu, and G. Quan, "Simulation study on the dynamic ventilation control of single head roadway in high-altitude mine based on thermal comfort," Adv. Civil Eng., vol. 2019, pp. 1-12, Jul. 2019. 
12. Li, K. Deb, Q. Zhang, and S. Kwong, "An evolutionary many-objective optimization algorithm based on dominance and decomposition," IEEE Trans. Evol. Comput., vol. 19, no. 5, pp. 694-716, Oct. 2015.

13. S. Wen, C. Huang, X. Chen, J. Ma, N. Xiong, and Z. Li, "Energy efficient and delay-aware distributed routing with cooperative transmission for Internet of Things," J. Parallel Distrib. Comput., vol. 118, pp. 46-56, Aug. 2018.

14. K. Gao, F. Han, P. Dong, N. Xiong, and R. Du, "Connected vehicle as a mobile sensor for real time queue length at signalized intersections," Sensors, vol. 19, no. 9, pp. 2039-2059, Nov. 2019.

15. E. Topal and S. Ramazan, "A new MIP model for mine equipment scheduling by minimizing maintenance cost," Eur. J. Oper. Res., vol. 207, no. 2, pp. 1065-1071, Dec. 2010.

16. Y. Jiang, G. Tong, H. Yin, and N. Xiong, "A pedestrian detection method based on genetic algorithm for optimize XGBoost training parameters," IEEE Access, vol. 7, pp. 118310-118321, 2019.

17. W. Gao, G. Li, Q. Zhang, Y. Luo, and Z. Wang, "Solving nonlinear equation systems by a two-phase evolutionary algorithm," IEEE Trans. Syst., Man, Cybern. Syst., early access, Dec. 20, 2020.

18. W. Gong, Y. Wang, Z. Cai, and S. Yang, A weighted bi-objective transformation technique for locating multiple optimal solutions of nonlinear equation systems," IEEE Trans. Evol. Comput., vol. 21, no. 5, pp. 697-713, Oct. 2017.

19. Y. R. Naidu and A. K. Ojha, "Solving multiobjective optimization problems using hybrid cooperative invasive weed optimization with multiple populations," IEEE Trans. Syst., Man, Cybern. Syst., vol. 48, no. 6, pp. 821-832, Jun. 2018.

20. Q. Gu, H. Xie, R. R. A. Issa, and C. Lu, "Location optimization with uncertainty for industrial project using discrete block model and spatial meshing algorithm," J. Comput. Civil Eng., vol. 33, no. 2, Dec. 2019, Art. no. 04018064.

21. S.M.. J. Rodriguez. Green Communication for 4G Wireless Systems. River Publishers; 2013

22. A.-M.B..N.N.. H.H. Downlink scheduling with economic considerations for future wireless networks. IEEE Trans Veh Technol 2009;58(2):835-824. doi:10.1109/TVT.2008.927039.

23. Y.-C.W..T.-Y. Tsai. A pricing-aware resource scheduling framework for lte networks. IEEE/AC

24. G.P.L.A.G..G.B.. R.F.. P. Camarda. Two-level downlink scheduling for real-time multimedia services in lte networks. IEEE Trans Multimedia 2011;13(5):1052-65. doi:10.1109/TMM.2011.2152381. 